\title{
Análisis de necesidades de aprendientes haitianos: diseño, validación y aplicación del instrumento
}

\author{
Gloria Toledo Vega, Francisco Quilodrán \\ y Libertad Korchabing
}

En este estudio se diseña, valida y aplica un instrumento de análisis de necesidades para implementar mejoras en la enseñanza-aprendizaje de español como lengua extranjera entre adultos haitianos en Chile. Su aporte consiste en la conformación de un set de ítems que exploran las necesidades, intereses y estilos de aprendizaje de la mencionada comunidad de aprendientes. El instrumento contempla siete dimensiones que permiten indagar sobre el conocimiento de la lengua meta, la valoración del español, las características del profesor ideal de ELE, la organización y el ambiente de la clase, los estilos de aprendizaje, la evaluación y la motivación para estudiar español entre los haitianos. Aplicado el análisis de necesidades a un grupo de 113 aprendientes, validamos el instrumento en cuanto a su consistencia interna, para que pueda ser aplicado a posteriores grupos de aprendientes haitianos que reciben clases de ELE en Chile. Finalmente, resumimos los resultados del análisis de los 113 informantes, aportando sugerencias didácticas de acuerdo a los datos aportados por nuestro instrumento.

Palabras claves: español como lengua extranjera, inmigrantes haitianos, análisis de necesidades, validación de instrumentos.

Needs analysis of Haitians learners: application, analysis, and validation of a questionnaire: In this study, a needs analysis instrument is designed, validated and applied to implement improvements in the teaching-learning of Spanish as a foreign language among Haitian adults in Chile. Its contribution consists of the creation of a set of items that explore the needs, interests, learning styles and strategies of the aforementioned community of learners. The instrument includes seven dimensions that allow us to inquire about the knowledge of the target language, the assessment of Spanish, the 
characteristics of the ideal SSL teacher, the organization and the classroom environment, the learning styles, the evaluation, and the motivation to study Spanish among Haitians. Once applied the needs analysis to a group of 113 learners, we validated it as an instrument in terms of its internal consistency, so that it could be applied to subsequent groups of Haitian learners who receive SSL classes in Chile. Finally, we summarize the results of the analysis of the 113 informants, providing didactic suggestions according to the data provided by our instrument.

Keywords: Spanish as a foreign language, Haitian immigrants, needs analysis, validation of instruments.

\section{Introducción}

Dadas las actuales circunstancias de inmigración en todo el mundo, las naciones en vías de desarrollo se han convertido en centros que atraen a diversos colectivos de inmigrantes. Este es el caso de Chile, cuya inmigración desde la década del 90 ha procedido de países latinoamericanos hispanohablantes, pero que hoy en día acoge a un número creciente de inmigrantes hablantes no nativos de español. Este nuevo escenario para la propagación de nuestra lengua supone desafíos en varios ámbitos, entre ellos, la enseñanza del español a las comunidades de hablantes no nativos y su inserción en la sociedad chilena. El doble desafío de inserción y de aprendizaje del nuevo idioma puede asumirse desde el mismo aprendizaje de la lengua, que como portadora de significados socioculturales es clave para encontrar trabajo, para especializarse y para insertarse en la cultura del medio local (Gutiérrez 2013).

Dentro de las comunidades hablantes no nativas de español que se han establecido en nuestro país, destaca la población haitiana que ha experimentado un crecimiento exponencial durante los últimos ocho años (Departamento de Extranjería y Migración de Chile 2018). La llegada de haitianos ha traído consigo personas de todas las clases sociales, con diferentes niveles de educación y variada formación profesional o académica. En cualquier caso, y al margen de su nivel socio-educacional, la mayor parte de esta comunidad se ubica en un segmento socioeconómicamente vulnerable cuando se establece en Chile. Esta situación dificulta que los haitianos accedan a clases de español pagadas, lo que a su vez complica su acceso a puestos de trabajo especializados y preserva un círculo de vulnerabilidad difícil de romper. Ante esto, diversas organizaciones han puesto a disposición de los haitianos clases de español como lengua extranjera, sin embargo, la oferta de estas clases se vale de 
recursos limitados en términos de salas de clases, material de estudio, horas de trabajo y capacitación de instructores, todos quienes hoy en día trabajan como voluntarios y optimizan de la mejor forma posible los pocos recursos con los que cuentan.

En el contexto recién mencionado participaron en nuestra investigación dos organizaciones que imparten clases de español como lengua extranjera (ELE): el Servicio Jesuita para Migrantes (SJM) y la Escuela y Fundación Jean Jacques Dessalines (JJD). El SJM opera con 115 voluntarios y ofrecen 27 cursos que se hacen principalmente en colegios durante el fin de semana en la tarde. Sus instructores trabajan con guías elaboradas por quienes tienen una mejor preparación en la instrucción de ELE, las cuales se complementan con guías de buenas prácticas y de inducción para los voluntarios. El SJM ofrece un curso de 13 sesiones, impartidas una vez por semana durante dos horas. Algunos voluntarios incorporan otro día de repaso o de reforzamiento en la semana para quienes tienen disponibilidad y mayores dificultades. El número total de estudiantes asociados al SJM bordea las 500 personas. En la Escuela JJD, en tanto, existen 22 voluntarios dedicados a la instrucción, de los cuales cinco de ellos elaboran material. La Escuela JJD funciona en un solo colegio, donde tienen 10 salas disponibles para cinco niveles, cada uno de los cuales tiene alrededor de 40 estudiantes. En total tienen 250 estudiantes en lista, con unos 200 que asisten con regularidad, una vez por semana durante 3 horas. Los pocos instructores que elaboran el material son quienes han recibido una formación en el área de ELE. Tanto en las clases del JJD como en las del SJM hay un estimado de $20 \%$ de deserción o rotación entre sus alumnos y, por lo mismo, lograr la motivación entre los estudiantes haitianos es una preocupación importante para ambas organizaciones.

Nuestro trabajo pretende colaborar con estas y otras organizaciones para mejorar sus clases de ELE y lograr la ansiada motivación de su alumnado haitiano. Para esto nos abocamos a la recogida de información respecto a las necesidades e intereses de los aprendientes, mediante el diseño y validación de un cuestionario de análisis de necesidades. La idea es facilitar un cuestionario pertinente en su validez y en su consistencia interna para la implementación de mejoras en la enseñanza-aprendizaje de adultos haitianos aprendientes de español como lengua extranjera. En el contexto chileno esta contribución es relevante, dada la falta de cuestionarios de análisis de necesidades para aprendientes de ELE que aporten a sus usuarios precisión para reportar conclusiones coherentes y contribuir efectivamente al mejoramiento de la enseñanza-aprendizaje de la lengua de acogida (Arancibia et al. 2016). 


\section{Lineamientos teóricos sobre el análisis de necesidades}

El análisis de necesidades (AN) nace intrínsecamente unido al diseño curricular, ya que es una actividad de desarrollo del currículo propiamente dicho (Yalden 1987: 42) y su práctica comienza a expandirse durante los años 70, como consecuencia del auge de la enseñanza centrada en el alumno. A fines de los 70 y a comienzos de los 80 , con hitos como la publicación de Un Nivel Umbral (Slagter 1979), el interés por los estudios de análisis de necesidades se fue extendiendo a cursos de segunda lengua o lengua extranjera en general. La importancia que se le dio al AN en esta época tiene que ver con una nueva perspectiva en la enseñanza, que comprende que no existen métodos o metodologías que sean la última palabra para enseñar o aprender lenguas extranjeras. En consecuencia, los enfoques centrados en las necesidades e intereses de los alumnos harán posible la motivación de estos y un mayor involucramiento en su proceso de aprendizaje (García Santa-Cecilia 2008: 87).

\subsection{Análisis de necesidades y definición de objetivos}

Prácticamente toda la literatura sobre análisis de necesidades enfatiza en que este proceso es esencial para el diseño e implementación de cursos de segunda lengua. En esta línea, Long (2005) llama a que la Lingüística Aplicada comprometa un esfuerzo para identificar generalizaciones sobre cómo conducir mejor los análisis de necesidades en poblaciones, sectores y condiciones específicos, que es justamente lo que queremos hacer en este trabajo con la población inmigrante haitiana en Chile. Un adecuado AN servirá para que los aprendientes sean ubicados correctamente en cursos, para diseñar programas y materiales, y para plantear los objetivos con un grupo particular en mente (Altman 1980; Berwick 1989; Norton y Toohey 2001; Long 2005). Los objetivos, guiados por un adecuado análisis de necesidades, establecen las intenciones pedagógicas del curso, fuerzan a los diseñadores del curso a ser realistas y facilitan la evaluación y la selección de materiales y actividades (Yalden 1987: 42). Este vínculo entre análisis de necesidades y objetivos no solo se remite a la fase inicial de un curso, ya que el AN permite modificar, orientar o ampliar los planteamientos iniciales. De hecho, los procesos evaluativos pueden considerarse como un AN continuo (Brown 1989), cuyo objetivo es depurar las ideas derivadas del análisis inicial para mejorar el curso en todas sus dimensiones y con todos sus actores: pro-

Lengua y migración / Language and Migration 12:1 (2020), 101-122

Edición impresa: ISSN 1889-5425. Edición en línea: ISSN 2660-7166. @ U Universidad de Alcalá 
fesores, estudiantes, diseñadores de cursos, autoridades del centro de estudios, etc. (Richterich y Chancerel 1980; Richterich 1983; Berwick 1989; Brindley 1989 y Robinson 1991).

En el contexto de la enseñanza-aprendizaje de inmigrantes haitianos en Chile, nuestro cuestionario busca determinar los objetivos que pueden plantearse en una clase de ELE. Considerando que los instructores voluntarios de español no tienen una mayor preparación en el área de enseñanza de segundas lenguas, el AN les aportará un eje sobre el cual articular las clases, atendiendo a lo que los aprendices efectivamente necesitan.

\subsection{Análisis de necesidades y motivación}

Además de ser una guía para la formulación de objetivos, y justamente a raíz de ello, el AN es un elemento esencial para favorecer la motivación de los aprendientes. $\mathrm{Al}$ respecto, existen cuatro conceptos de motivación con los cuales los profesores de segundas lenguas (SL) suelen estar más familiarizados, los dos conceptos clásicos de motivación instrumental e integradora de Gardner y Lambert (1959) y la motivación extrínseca e intrínseca. La motivación instrumental se asocia a intereses prácticos como el ámbito laboral, y la motivación integradora se relaciona con intereses de tipo sociocultural relacionados con la comunidad meta. La motivación extrínseca alude a intereses externos como aprobar un curso o un examen o satisfacer las demandas de los padres para aprender un nuevo idioma. La contraparte intrínseca, en cambio, nace de un interés personal por aprender la segunda lengua (Muñoz 2000). A partir de estos cuatro conceptos se desarrollaron estudios psicológicos sobre la motivación que han determinado distintas variantes de los cuatro tipos recién descritos. Según Muñoz (2000), en un contexto formal de aprendizaje de segunda lengua los factores que pueden incidir en la motivación son la actitud, la edad, las horas de clase, el rendimiento lingüístico, los factores afectivos, los efectos del profesor, las tareas y los materiales. Desde otro enfoque, Norton (1995) propone reemplazar el concepto de motivación por el de inversión. De acuerdo a esta autora, cuando se aprende una SL se hace una inversión cultural mediada por los distintos interlocutores y la relación que los aprendientes tengan con estos. En este sentido, la motivación es cambiante al igual que la identidad de los aprendientes de una lengua. Un trabajo que va en esta misma línea es el de García-Romeu (2006), quien investigó los resultados de una encuesta de AN para promover la motivación e implicación de los estudiantes y estimular el aprendizaje cooperativo, advirtiendo que la motivación no es lineal, ya que está vinculada con la percepción de cada aprendiente y con el valor que cada uno percibe respecto a la actividad concreta que tiene que realizar. 
En cualquiera de los casos mencionados, el AN constituye el mejor medio para conocer qué factores determinan la motivación de los aprendientes y, por lo mismo, es importante para diseñar los cursos e involucrar a los alumnos en su aprendizaje (Hawkey 1980; Mc Donough 1984; Berwick 1989; Ridell 1991; Savage y Stoner 1992). El análisis de necesidades es un elemento vinculado estrechamente con un diseño curricular flexible y abierto, centrado en el alumno y, por lo tanto, de carácter comunicativo. Un currículo de esta naturaleza, ya sea que esté centrado en el proceso o en el producto, permitirá incorporar objetivos relacionados con la motivación de nuestros estudiantes, información que conoceremos mediante la recogida de información objetiva y subjetiva sobre quiénes son y cómo aprenden.

En relación con nuestro estudio, es frecuente escuchar entre los instructores voluntarios que la motivación de los estudiantes haitianos es de carácter instrumental y extrínseca, sin embargo, una investigación de Toledo (2016) muestra que la motivación de los aprendientes haitianos tiene mucho que ver con la integración de estos en la sociedad de acogida. Nuestro estudio pretende clarificar esto con preguntas validadas que orienten mejor respecto a qué lleva a estos sujetos a aprender español.

\subsection{Información objetiva y subjetiva en un análisis de necesidades}

La información que puede recabar un AN puede ser objetiva y/o subjetiva. Richterich (1983) distingue entre necesidades objetivas que se obtienen a partir de datos concretos del aprendiente al principio del curso, y necesidades subjetivas de tipo cognitivo, afectivo, relacionadas con la personalidad, confianza, actitud, deseos y expectativas del aprendiente, que pueden implementarse durante el desarrollo del curso. La información subjetiva refleja las percepciones de los aprendientes sobre qué deben aprender y cómo, y revela preferencias sobre estilos de aprendizaje. El Portafolio europeo de las lenguas es un instrumento que recoge este tipo de información (Consejo de Europa 2001) que es útil para los aprendices, pues les sirve para tomar conciencia de su aprendizaje, y para los docentes, que reciben información sobre la autopercepción de su alumnado. Indagar sobre las necesidades subjetivas puede servir para alinear las agendas de los aprendientes y sus profesores, que no siempre van por la misma ruta.

El cuestionario que diseñamos y validamos indaga las necesidades subjetivas de los aprendientes haitianos, lo cual adquiere relevancia si tomamos en cuenta la inexperiencia de muchos de sus instructores 
voluntarios. Una buena parte de estos instructores son profesores de niños o adolescentes en la escuela, que estiman que un ambiente de clase centrado en el profesor, estricto y silencioso es fundamental para el éxito de la clase. No obstante, estas características no siempre rinden buenos frutos cuando queremos promover la comunicación entre adultos en el aula.

La información objetiva, en tanto, puede conseguirse a partir de pruebas de diagnóstico que determinan lo que sabe el aprendiente y/o lo que necesita saber para alcanzar un nivel determinado en la lengua objeto. Además del nivel de lengua, la información objetiva apunta a datos como la edad, la lengua materna, el grado de competencia en otras lenguas extranjeras, el tiempo de aprendizaje de la lengua objeto y/o el tiempo de inmersión en la comunidad meta. Un nivel umbral, en su versión al español de Slagter (1979), es un modelo clásico para el análisis de necesidades de este tipo.

West (1994) distingue entre el AN de situaciones meta (lo que necesita saber el aprendiente para desenvolverse en una determinada situación); el AN de deficiencias del alumno (que considera las lagunas de conocimiento de los aprendientes); y el análisis de medios (recursos disponibles y actitudes de la sociedad meta hacia los aprendientes). Los dos primeros tipos de análisis responden a necesidades objetivas y las dos últimas variantes a necesidades subjetivas. Hutchinson y Waters (1987) se refieren a "necesidades meta" (relacionadas con objetivos pedagógicos) y "necesidades de aprendizaje" (relacionadas con los programas). Estos autores subcategorizan las necesidades meta en: (i) necesidades propiamente tal: lo que necesita saber el aprendiente para funcionar en una situación determinada; (ii) carencias: la discrepancia entre las necesidades y lo que en realidad sabe el aprendiente; y (iii) deseos: lo que quiere aprender o siente que necesita aprender el estudiante y que a veces no concuerda con las percepciones del profesor. Bajo cualquier perspectiva, un elemento central para cubrir las necesidades objetivas es la evaluación, sobre la cual no existe una política clara en las organizaciones que imparten clases de ELE a inmigrantes en Chile. La mayoría de los instructores voluntarios prefiere no evaluar, por distintas razones: falta de conocimientos para preparar evaluaciones en ELE, la sensación de que una mala nota les restará motivación a sus alumnos, la dificultad para aplicar y dejar un registro sistemático de la evaluación formativa, entre otros factores. Para nosotros es importante saber qué piensan los alumnos al respecto y por eso incluimos ítems sobre evaluación en nuestro cuestionario. La evaluación sirve para registrar necesidades objetivas, para seguir y fomentar el progreso de los alumnos y para dar un carácter de mayor formalidad a la clase, entre otras razones por las cuales habría que considerarla. 


\subsection{Aproximación al proceso o al producto}

En cuanto a la selección de información que busca un determinado análisis, Nunan (1988) ejemplifica con los datos requeridos por Munby (1978) y los suyos propios (Nunan y Burton 1985) para reflexionar acerca de la orientación de los AN. La información que recaba Munby de los aprendientes incluye: edad, lengua materna, manejo de la lengua objeto y de otras L2, fin específico de estudio, lugar de desempeño, características del lugar de desempeño, potenciales interactuantes en dicho lugar, propósito del empleo de la lengua objeto, forma en que llevará a cabo la comunicación (cara a cara, por teléfono...), tipo de dialecto que empleará y eventos comunicativos en los que participará. El AN de Nunan y Burton (1985), en tanto, incluye información relacionada con el nombre, la edad, el tiempo en el país de la lengua meta, la nacionalidad, la educación, la ocupación, el nivel de la lengua meta, los recursos de la lengua materna, las metas de aprendizaje, la disponibilidad, la motivación y el ritmo de aprendizaje de los aprendientes. Con estos datos Nunan propone reflexionar sobre la compatibilidad de varios estudiantes de distintas características en un mismo curso y determinar si esta información conduce al diseño de un curso orientado al proceso o al producto. En relación con lo anterior, el análisis de Munby parece diseñado para un curso de propósitos específicos, orientado al producto, mientras que la información recabada por Nunan y Burton tiende más a influir en el proceso de enseñanza-aprendizaje. Nunan (1988: 79) sugiere reflexionar sobre qué podemos hacer si, por ejemplo, el pre diseño de nuestro currículo se encauza al juego y resulta que nuestros alumnos prefieren un contexto de clases tradicional más 'serio`.

La respuesta a las reflexiones que sugiere Nunan está en el manejo de un currículo flexible, es decir, un tipo de curso que tenga una estructura de base lo suficientemente abierta para incorporar o sacar contenidos y/o objetivos de acuerdo a las necesidades de los estudiantes (Yalden 1987). El instrumento que diseñamos y validamos tiende a explorar más que nada el proceso, pues busca una forma de ayudar a los instructores a llevar la clase de la forma más ajustada posible a los intereses de sus alumnos, pero tomando siempre en cuenta que la variedad etaria, educacional, laboral y social en un solo curso puede ser muy amplia, lo cual descarta una aproximación a propósitos demasiado específicos.

\subsection{Identificación de estrategias y estilos de aprendizaje}

A partir de los años 90 y durante la primera década del presente siglo, el análisis de necesidades abrió paso a la identificación de las estrate-

Lengua y migración / Language and Migration 12:1 (2020), 101-122

Edición impresa: ISSN 1889-5425. Edición en línea: ISSN 2660-7166. ㄷ Universidad de Alcalá 
gias y estilos de aprendizaje. Esta tendencia se explica por la creencia de que los aprendientes que desarrollan habilidades para aprender a aprender explotarán mejor las oportunidades de las clases y estarán mejor preparados para el aprendizaje fuera del aula (Wong y Nunan, 2011: 144).

García Santa-Cecilia (2008) observa las necesidades desde dos perspectivas: (1) la información sobre el individuo (edad, personalidad, aptitud, inteligencia y motivación), la información menos evidente sobre estilos y estrategias cognitivas, grado de ansiedad, nivel de autoestima y grado de disposición a tomar riesgo; y (2) la información sobre los estilos de aprendizaje de los alumnos. En la misma línea, Willing (1988) define el estilo de aprendizaje como las características que permiten agrupar a las personas en tipos a lo largo de una escala descriptiva. Los estilos no deben confundirse con las estrategias de aprendizaje, en tanto las últimas son formas específicas de lidiar con tareas en contextos particulares, mientras que los estilos son una aproximación más general al aprendizaje de la lengua (Cohen 2003; Oxford 2003). Jones et al. (1987) vinculan ambos conceptos al señalar que los estudios revelan más eficacia y efectividad entre los aprendientes que están conscientes de los procesos que subyacen a su aprendizaje, lo cual les permite alinear estrategias y metas para aprender.

Sobre la eficacia en el aprendizaje, Benson (2001) y Benson y Nunan (2005) sostienen que los aprendientes más eficaces conciben la lengua objeto como una herramienta de comunicación más que como un objeto o meta de estudio. En una línea similar, Wong y Nunan (2011) señalan que los estudiantes más eficaces son los más comunicativos y autónomos. Los estudiantes menos efectivos, por otra parte, están más orientados a la autoridad y prefieren una secuencia estructurada de aprendizaje. No obstante, queda la duda de si los estudiantes más eficaces son así porque emplean más tiempo comunicándose autónomamente en la lengua meta, o si acaso el hecho de pasar más tiempo empleando la lengua meta se debe a que son más competentes en esta. De cualquier forma, la eficacia puede explicarse por una mayor autonomía de los estudiantes, la que a su vez puede incrementarse haciéndolos conscientes respecto a cuáles son las mejores estrategias de aprendizaje para ellos (Ungureanu y Georgescu, 2012).

Nuestro cuestionario aborda preguntas para conocer si el estilo de aprendizaje de los haitianos se inclina más por aprender para comunicarse o aprender para acumular conocimientos. Sondear esto es importante dado que, como mencionamos, el estilo de enseñanza de quienes son profesores de escuela primaria o secundaria en Chile privilegia la revisión de contenidos por sobre el trabajo y la práctica con ellos. De hecho, "pasar toda la materia" se establece como una prioridad por las 
mismas autoridades educativas a nivel estatal o de dirección particular de la escuela.

Dado que el empleo de estrategias de aprendizaje se vincula con contextos más específicos, no lo consideramos mayormente en nuestro cuestionario, que aborda las respuestas de 113 informantes de distintos cursos de ELE, sin embargo, creemos que en el contexto de una clase particular sería muy provechoso obtener información al respecto.

Ahora bien, nuestro instrumento no pretende, ni podría pretender, asociar a toda una comunidad de hablantes no nativos con un solo estilo de aprendizaje o al uso de determinadas estrategias. Entendemos que las clases, los contextos de clase, los individuos que enseñan y que aprenden pueden ser muy diferentes. En un estudio con inmigrantes, Willing (1994) establece una hipótesis que relaciona las estrategias preferidas de los aprendientes con características biográficas tales como la lengua materna o el nivel de educación. Su propia investigación, sin embargo, le sirvió para rechazar tal hipótesis, ya que el favoritismo por ciertas estrategias parece estar más relacionado con las personalidades y estilos cognitivos particulares de los aprendientes, que con la etnia o nivel educacional de estos. En contextos en que las necesidades son generales y diversas es importante que los instructores lleven a cabo una reflexión crítica de los cuestionarios de análisis de necesidades, para identificar tareas genéricas que satisfagan las demandas de todos, pero que también cubran necesidades más específicas (Serafini et al. 2015a; Serafini y Torres 2015b; Todea y Demarcsek 2017). Evidentemente, en clases menos numerosas se podrá trabajar el autoconocimiento y manejo de las estrategias particulares de aprendizaje, además de incorporar fines específicos sobre los cuales, en cualquier caso, los docentes deberán preguntarse si lo que enseñan es adecuado o no para sus estudiantes (Catalá y Camarero 2017).

Para el diseño de nuestro cuestionario tomamos en cuenta principalmente los criterios de Willing (1994) que identificó cuatro estilos de aprendizaje. El primer estilo es el comunicativo, a quienes les gusta aprender mirando o escuchando a los hablantes nativos, practicar con amigos en la lengua objeto y usar la lengua objeto fuera de clases. Otro estilo es el analítico, de quienes prefieren estudiar gramática, emplear el libro de clases, estudiar solos, encontrar sus propios errores y trabajar en problemas planteados por el profesor. El tercer estilo es aquel orientado a la autoridad, estos aprendientes prefieren que el profesor les explique todo, les gusta escribir todo en un cuaderno, estudian gramática y aprenden leyendo. Por último, Willing identifica el estilo concreto, que caracteriza a quienes les gustan los juegos, trabajar con imágenes, películas, videos y audios, hablar en parejas y practicar la lengua meta fuera de clases. 
Ante el amplio panorama de estrategias y estilos de aprendizaje nuevamente surge la pregunta de cómo proveer la metodología adecuada a los diversos estilos que conviven en una clase. Como señala Gnutzmann (2005), no hay un perfil único del buen aprendiente y las diferencias entre ellos son numerosas en términos de estrategias. Tudor (1996) ofrece dos caminos: seleccionar metodologías que coincidan con las preferencias de los alumnos o enfrentar a los alumnos con formas de estudio y trabajo que no concuerdan con sus preferencias y estilos, lo cual les servirá para ampliar sus opciones de aprendizaje. Una tercera vía es que la pedagogía tenga un estilo neutral y se enfoque en animar a los aprendientes a descubrir y trabajar su propio estilo de aprendizaje (Christison 2003). En efecto, "la labor del profesor en relación con los estilos de aprendizaje de sus alumnos consiste [...] en el desarrollo de actitudes individuales y de grupo que permitan crear en clase un clima propicio para el aprendizaje” (García Santa-Cecilia 2008: 98).

\section{Metodología}

\subsection{Instrumento}

Para analizar las necesidades de aprendientes de segundas lenguas es posible emplear cuestionarios o entrevistas. Una ventaja de las entrevistas abiertas es que pueden identificar las preguntas más relevantes para un análisis de necesidades. Los cuestionarios, por su parte, asumen que las preguntas que se formulan son las correctas, pero en comparación con las entrevistas la ventaja de los cuestionarios es que pueden ser administrados en grupos, por terceras partes, incluyen a más informantes, son fáciles de organizar, facilitan el anonimato y previenen los sesgos del investigador (Long 2005: 38).

Para realizar un buen cuestionario de AN tomamos en cuenta las condiciones de Long (2005): evitar preguntas que conduzcan a respuestas cerradas (sí/no); evitar el uso de lenguaje teórico o muy complejo; evitar hacer preguntas tendenciosas que den por sentada información; por ejemplo ¿̇ómo se siente aprendiendo una lengua tan difícil como el español?; evitar la ambigüedad y/o la abstracción en la formulación de las preguntas; y evitar hacer preguntas amenazantes o irrelevantes. Para facilitar el análisis, las respuestas y la agilidad en la cumplimentación, nuestro cuestionario contempla respuestas por escala: muy de acuerdo, de acuerdo, en desacuerdo y muy en desacuerdo para 75 afirmaciones distribuidas en ocho dimensiones: (1) conocimiento de la lengua meta, con 7 preguntas; (2) valoración del español, con 5 preguntas; (3) características del profesor, con 14 preguntas; (4) organización de la clase y 
actividades, con 13 preguntas; (5) ambiente de la clase, con 6 preguntas; (6) estilos de aprendizaje de ELE, con 15 preguntas; (7) evaluación, con 8 preguntas; y (8) motivación para estudiar español, con 7 preguntas. Además, incluimos 9 temas para que los aprendientes marcaran los que eran de su interés: trabajo, cultura (arte, literatura, cine...), vida diaria, colegio/ escuela, transporte, comercio, vida en comunidad, relaciones sociales y familiares, y otro (especificar).

Conforme a estas dimensiones recogimos información de necesidades objetivas (datos sobre edad, sexo, manejo de la lengua objeto y de otras lenguas y tiempo de estadía en Chile), subjetivas (necesidades cognitivas, afectivas, actitudinales, volitivas, estilos y estrategias de aprendizaje).

\subsection{Validación de contenido a través de expertos}

Finalizado el diseño del instrumento de 75 ítems, se realizó un proceso de validación. En este trabajo empleamos dos tipos de validez: de contenido y de estructura interna. La primera consistió en conocer, a través de jueces expertos en el tema, las posibles fallas en la redacción de los ítems de cada dimensión (Sireci y Padilla 2014). Para esto contamos con tres expertos: un psicólogo especialista en educación de adultos, un experto en el tema de validación de instrumentos psicométricos y un profesor de español como lengua extranjera con vasta experiencia. A los tres expertos se les pidió clasificar los ítems en tres grupos y puntuarlos de la siguiente manera: (1) cambiar definitivamente, (2) podría necesitar un cambio y (3) no es necesario cambiar. Una vez obtenidas las puntuaciones de los expertos se procedió a definir los cambios. Ninguno de los ítems obtuvo una puntuación de 1, pero 8 obtuvieron una puntuación 2 por parte de uno de los expertos, con lo cual se decidió hacer algunas modificaciones. El resto de ítems fue calificado con puntuación 3. Los cambios que se realizaron consistieron en modificar algunos ítems que no eran específicos y mejorar la redacción de otros para que fueran más fáciles de entender por los encuestados.

$\mathrm{El}$ instrumento fue creado en español y validado en esa lengua, sin embargo, debido a que sería cumplimentado por aprendientes haitianos con distintos niveles de español, se hizo una traducción del cuestionario al creole. Para este proceso se solicitó el trabajo de dos traductores creole-español. Ambos traductores eran hablantes nativos de creole y dominaban el español como segunda lengua. No fue posible localizar traductores nativos de español que manejaran el creole, por lo tanto, se decidió trabajar con estos dos traductores, si bien lo ideal es que el proceso de traducción cuente con hablantes nativos de los pares de idiomas con los que se trabaja, es decir, creole y español (Harkness y Schoua- 
Glusberg, 1998). El procedimiento fue el siguiente: uno de los traductores tradujo el cuestionario al creole y luego el otro traductor, que tenía mayor conocimiento de español, tradujo desde el creole al español. Posteriormente se revisó que los ítems en la segunda versión fueran similares a los de la versión original, lo que ocurrió en todos los casos.

Para el segundo tipo de validación calculamos la confiabilidad del cuestionario, cuyo resultado completo fue de un alfa de 0.888 , cifra que se interpreta como un alfa óptimo (George y Mallery 2003), especialmente en caso de investigaciones exploratorias como esta (Gliem y Gliem 2003). En la tabla 1 se puede observar que los ítems 3, 5, 10, 11, $27,33,66,73$ y 75 aumentan la confiabilidad del cuestionario, pues ayudan a la consistencia interna. Los ítems 44, 46, 42 y 60, afectan la confiabilidad, aunque no de manera importante.

\begin{tabular}{|c|c|c|}
\hline Ítem & \multicolumn{1}{|c|}{ Varianza de escala } & Alfa de Cronbach \\
\hline 3 & 353,169 &, 889 \\
\hline 5 & 354,321 &, 890 \\
\hline 10 & 353,125 &, 889 \\
\hline 11 & 349,608 &, 889 \\
\hline 27 & 345,972 &, 889 \\
\hline 33 & 349,935 &, 889 \\
\hline 44 & 332,394 &, 884 \\
\hline 46 & 336,722 &, 884 \\
\hline 52 & 331,757 &, 883 \\
\hline 60 & 336,857 &, 884 \\
\hline 66 & 347,741 &, 889 \\
\hline 73 & 349,575 &, 889 \\
\hline 75 & 345,495 &, 889 \\
\hline
\end{tabular}

Tabla 1. Confiabilidad del cuestionario

Validado el instrumento lo aplicamos a 113 aprendientes haitianos que tomaban clases en la Escuela Jean Jacques Dessalines y en una de las sedes de enseñanza de ELE para haitianos, perteneciente al Servicio Jesuita para Migrantes. Si bien entregamos el cuestionario escrito en creole, le pedimos a un traductor que fuera leyendo los ítems para favorecer la agilidad en la cumplimentación, para solucionar posibles dudas y para que las personas no alfabetizadas también pudieran cumplimentar el instrumento. En total, este proceso se extendió por 40 minutos. 


\subsection{Características de los informantes}

Los participantes en este estudio forman parte de una muestra no probabilística de 113 estudiantes haitianos adultos. La muestra estuvo conformada por 68 hombres y 37 mujeres, proporción que refleja bien la distribución por sexo de la población haitiana en Chile. El rango de edades de los participantes fluctuaba entre los 20 y 50 años. Sus niveles de español eran variables, pues les pedimos su participación a aprendientes de niveles A1, A2, B1 y B2. En cuanto a la cantidad de años en Chile, un $62 \%$ llevaba menos de un año en el país y un $23 \%$ llevaba entre uno y dos años. Quienes manejaban otros idiomas generalmente tenían conocimientos de francés e inglés y eran personas con mayor nivel de educación, muchos de ellos con educación superior.

\section{Análisis de los resultados}

Para explicar con claridad los resultados del cuestionario distribuimos la información obtenida en cada dimensión del instrumento. De cada dimensión relevamos los ítems que obtuvieron más de 50\% de selección, pues ellos reflejan los datos más importantes para la mayoría de la población participante en este estudio. Grosso modo, los ítems 1, 7 y 9 aportan información sobre necesidades objetivas de los aprendientes, en tanto los ítems 2, 3, 4, 5, 6, 7 y 8 aportan datos sobre necesidades subjetivas.

En la dimensión sobre conocimiento de la lengua meta (7 preguntas), la mayoría de los haitianos evalúa mejor sus destrezas receptivas, de esta forma, un $58,8 \%$ está de acuerdo con que entiende cuando les hablan en español y un 53,1 \% está de acuerdo con que entiende textos escritos en esta lengua. Esta dimensión puede aportar pistas sobre los objetivos que se pueden considerar en el curso de ELE. En este caso, parece necesario incorporar la práctica de las destrezas productivas como un objetivo central.

En la dimensión sobre valoración del español (5 preguntas) una gran mayoría está muy de acuerdo respecto a que esta lengua es muy importante para ellos $(80,5 \%)$ y a que disfrutan aprender esta lengua $(78,8 \%)$. En otras palabras, la valoración del español por parte de los haitianos es alta. Una entrevista con los aprendientes o un grupo focal con sus instructores podría aclarar si esta valoración responde solo a una necesidad instrumental o a un factor más emotivo o cultural respecto a nuestra lengua. De cualquier forma, incluimos dos afirmaciones para saber si el aprendizaje del español por parte de los haitianos se vinculaba más a una motivación instrumental: "Estudio español solo porque es necesario" y

Lengua y migración / Language and Migration 12:1 (2020), 101-122

Edición impresa: ISSN 1889-5425. Edición en línea: ISSN 2660-7166. @ U Universidad de Alcalá 
"Uso el español solo cuando es muy necesario". La primera afirmación cuenta con un no despreciable $40,7 \%$ de selección y la segunda solo con $27,4 \%$.

La dimensión sobre las características del profesor ideal de ELE (14 preguntas) se relaciona con los estilos de aprendizaje de los aprendientes haitianos y muestra que un $81,4 \%$ de los informantes están muy de acuerdo en preferir un profesor que dirija la clase para que los alumnos solo escuchen. Un 67,3\% está muy de acuerdo en que el profesor debe tener ordenada y planificada su clase completamente. Por otro lado, un $64,6 \%$ está muy de acuerdo con que el profesor sea cercano a sus alumnos. Del mismo modo, un 61,1 \% considera que no es necesario que el profesor corrija los errores. Un 52,2\% está muy de acuerdo con que les gusta que el profesor a veces improvise y un 50,4\% está muy de acuerdo en preferir que el profesor hable solamente en español, evitando el creole u otra lengua vehicular en clase. Por último, un $49,6 \%$ está de acuerdo con que es mejor que el profesor tome todas las decisiones. Si bien este porcentaje no refleja la mayoría absoluta de los participantes en el cuestionario, nos pareció importante indicarlo por cuanto muestra un estilo más orientado a la autoridad (Willing 1994), reflejado también en el $81,4 \%$ que espera que el profesor dirija la clase sin que los estudiantes hablen. Considerando estos datos, parece ser que los alumnos esperan el control del profesor, si bien admiten que este a veces improvise y sea cercano a sus alumnos. Esto podría traducirse en una expectativa de relación vertical profesor-alumno en la que, no obstante, el profesor ha de ser cercano y ha de estar atento a no exponer demasiado a sus alumnos con correcciones o reprimendas (por atrasos).

La dimensión sobre organización de la clase y actividades (13 preguntas) también tiende a explorar los estilos de aprendizaje. Al respecto, los informantes están muy de acuerdo con que les gusta leer textos para la clase $(59,3 \%)$, lo que es consistente con el $49,6 \%$ que declara que sí tiene tiempo para hacer tareas en casa. Un 58,4\% de los aprendientes declara aburrirse cuando se hacen las mismas actividades siempre y un $51,3 \%$ cree que es provechoso trabajar en grupos. Por otra parte, un $54 \%$ de los aprendientes cree que los atrasos por parte de los alumnos deben ser aceptables por el profesor, sin embargo, una pequeña mayoría de un $32,7 \%$ estima que es molesto para la clase si un alumno llega tarde. La preferencia por el trabajo en grupos pareciera equilibrar un poco la tendencia a la autoridad que muestran los participantes en este cuestionario. Lo anterior nos lleva a considerar lo siguiente: un estilo orientado a la autoridad no tiene por qué descartar las necesidades comunicativas de un grupo de aprendientes. En este sentido, tiene sentido lo que señala Gnutzmann (2005) respecto a que no existe un perfil único del buen aprendiente. 
En la dimensión relacionada con el ambiente de la clase (6 preguntas) parece haber cierta contradicción, pues una mayoría de $76,1 \%$ está muy de acuerdo con que la sala de clases debe ser un lugar para relajarse y pasarla bien, mientras un $68,1 \%$ está muy de acuerdo con que el ambiente de clases debe ser disciplinado. De igual forma, a un 48,7 por ciento le gusta reírse y divertirse en la sala de clases, aunque un $43,4 \%$ estima que el ambiente de clases debe ser serio y de concentración. Frente a esto, la alternativa para el instructor sería conseguir un equilibrio donde exista una clara separación entre los momentos para estar concentrado (actividades de aprendizaje y conceptualización) y momentos de producción oral, que pueden propiciar un ambiente más distendido. Esta dimensión y la anterior pueden estar relacionadas en el sentido de que ambas muestran una necesidad de equilibrar la clase, dejando siempre dicho equilibrio en manos de un instructor que establezca distinciones claras respecto a las distintas actividades que se incluyen en la clase: actividades lúdicas, actividades de concentración, actividades de grupo, actividades receptivas y/o productivas.

Respecto a la dimensión sobre estilos de aprendizaje de ELE propiamente tal, que incluye también la preferencia por ciertas estrategias (15 preguntas), los resultados más importantes corresponden a la elección de "muy de acuerdo". En esta alternativa, un 72,6\% cree que es mejor practicar lo que se aprende. Un 71,7\% estima que es muy importante tener un apoyo escrito de las reglas morfosintácticas del español, con esquemas y explicaciones. Un $68,1 \%$ prefiere no arriesgarse a equivocarse cuando usa el español, de tal forma que su producción se genera cuando están seguros de hacerlo bien. Esto último parece consistente con que a los haitianos no les guste que corrijan sus errores y, por otra parte, el hecho de que estimen mejor sus destrezas receptivas por sobre las productivas. Un $65,5 \%$ cree que aprende mejor si el profesor explica las reglas claramente y luego las aplican. Un 54,9\% prefiere escuchar a hablantes nativos de español para aprender esta lengua. Un 51,3\% señala que aprende palabras nuevas conversando o mirando la TV y un 50,4\% declara que aprende palabras nuevas cuando las lee. En resumen, puede sostenerse que para estos aprendientes la práctica es importante, sin embargo, pareciera ser que esa práctica tiende más a la comprensión escrita y oral más que a la conversación. Dado que estos aprendientes identifican sus puntos más débiles en ELE con las destrezas productivas, valdría la pena tomar el camino de enfrentar a estos alumnos con formas de estudio y trabajo que no concuerdan plenamente con sus estilos (Tudor 1996).

La dimensión relativa a la evaluación (8 preguntas) se relaciona con las necesidades de aprendizaje objetivas de los alumnos. Aquí, un 61,3\% estima que no es necesario ponerles notas a los estudiantes, si bien un $59,3 \%$ estima que las evaluaciones les sirven para aprender de sus erro- 
res. Valga hacer notar que es distinto evaluar a poner notas; los estudiantes probablemente no quieren calificaciones, pero sí quieren evaluaciones y retroalimentación. No obstante lo anterior, un 49, 6\% de informantes considera necesario que los progresos de los alumnos sean evaluados con notas, lo que no corresponde a la mayoría de nuestros informantes, pero sí a un porcentaje importante. Esta contradicción le deja al instructor la alternativa de que las evaluaciones sean de tipo conceptual. Lo importante es que no se descarte la evaluación, porque estos aprendientes encuentran un valor en la recepción de retroalimentación. Por otra parte, es factible pensar que el hecho de que no les gusten las evaluaciones se relacione con su preferencia por no ser corregidos. No debemos olvidar que se trata de aprendientes adultos, que no tienen la misma tolerancia que los niños a cometer errores o a que les corrijan. Sobre la forma en que prefieren ser evaluados, un $52,2 \%$ se inclina por las evaluaciones escritas (aunque este es el único ítem en que solo están "De acuerdo" y no "Muy de acuerdo"). No obstante, un 50, 4 prefiere evaluaciones orales, lo que implica que el profesor debe optar por ambas en igual medida.

En lo que respecta a la motivación de los haitianos para estudiar español (7 preguntas), que corresponde a la última dimensión del cuestionario, aparece una sola selección mayoritaria que revela que un $67,3 \%$ de los informantes estudia español para que les vaya mejor en su trabajo y/o estudios, lo que es consistente con una motivación de carácter instrumental y concuerda con las elecciones de temas que les pedimos al final del cuestionario y que revisamos a continuación. Estos resultados contradicen en cierta medida a los de Toledo (2016), quien en un análisis de necesidades sobre funciones comunicativas descubrió una motivación de carácter integradora entre los aprendientes haitianos de ELE.

Por último, incluimos 9 temas para que los aprendientes señalaran aquellos de su interés: trabajo, cultura (arte, literatura, cine...), vida diaria, colegio/ escuela, transporte, comercio, vida en comunidad, relaciones sociales y familiares y otro (especificar). El tema más elegido fue el de trabajo (84 selecciones); seguido por el colegio/ escuela (71 selecciones); las relaciones sociales y familiares (68\%); la cultura y la vida diaria $(64 \%)$ y, finalmente, la vida en comunidad (63\%). El ítem menos votado fue "otro" y quienes lo seleccionaron no especificaron a qué "otro" se referían. El siguiente tema menos seleccionado fue "comercio" (27 selecciones), seguido por "transporte" con 35 selecciones.

\section{Conclusiones}

Vista la necesidad de aportar en la capacitación de instructores voluntarios de ELE para la población haitiana, elaboramos un análisis de nece- 
sidades que contempla ocho dimensiones tendientes a determinar cómo y qué enseñar en ELE (proceso, principalmente, y producto). Tras el diseño del cuestionario, se validó este instrumento con tres expertos y luego de forma estadística, obteniendo un alto porcentaje de confiabilidad. Se tradujo el cuestionario al creole y se aplicó a una población de 113 aprendientes haitianos.

El análisis de los resultados arrojados por los cuestionarios se dispuso de acuerdo a ocho dimensiones, cuya consideración es relevante en la enseñanza de español como lengua extranjera o segunda lengua. En efecto, el análisis de necesidades es un elemento estrechamente vinculado con el diseño curricular, que propiciará la motivación de nuestros aprendientes en tanto sea un currículo flexible y abierto, centrado en el alumno y, por lo tanto, de carácter comunicativo.

Tras la aplicación del instrumento que diseñamos y validamos, el análisis de su aplicación revela que la mayor parte de los aprendientes haitianos siente más confianza en sus habilidades lingüísticas receptivas (comprensión oral y escrita) que en las productivas (hablar y escribir). Este punto nos indica que es necesario trabajar la escritura (entre quienes están alfabetizados), pero especialmente la producción oral en clases.

La gran mayoría de estos aprendientes manifiestan un alto grado de valoración del español, no obstante, habría que esclarecer si esa valoración se basa en motivaciones de tipo instrumental o cultural. Si la motivación de nuestros aprendientes es primordialmente instrumental, habrá que centrar las clases de ELE en los ámbitos laborales y/o de estudios, sin embargo, nos parece necesario aclarar este punto con entrevistas personales por parte de los profesores en cada una de sus clases.

En cuanto al profesor de ELE, las inclinaciones de nuestros informantes los acercan a un estilo de aprendizaje orientado a la autoridad, pero a una autoridad cercana y no condenatoria (que no es lo mismo que permisiva). El profesor será quien ponga las reglas, pero deberá tener especial cuidado al momento de mantener un ambiente con bajo filtro afectivo en su clase, evitando exponer a sus alumnos al error, o bien, haciendo lo posible para que sus estudiantes pierdan el miedo a cometer errores.

Es revelador que nuestros informantes manifiesten que les gustaría hacer tareas, pero habría que comprobar esto en la práctica, pues nuestra experiencia docente nos ha mostrado que las tareas, si no son evaluadas, no siempre son realizadas por los estudiantes (sin importar su procedencia, etnia, nivel de lengua o género). Respecto a la evaluación, la mayoría de los informantes declaró que sí quiere ser evaluado, sin embargo, dado que varios declararon que las notas no son necesarias, sería conveniente emplear una evaluación de tipo conceptual, tanto oral como escrita, pero con mayor énfasis en la última. En cualquier caso, la evaluación debe ser entendida como una retroalimentación y un análisis 
de necesidades continuo respecto a las insuficiencias objetivas de los estudiantes (Brown 1989). A nuestro juicio, un curso de ELE no debiera descartar esta opción, es más, debiera trabajar en buscar o confeccionar rúbricas que les permitan a los docentes entregar la mejor retroalimentación posible y la mayor fiabilidad respecto a sus evaluaciones.

En el ámbito de las clases, los aprendientes haitianos parecen inclinarse por un equilibrio entre diversión y seriedad. Ese punto es interesante por cuanto varios profesores que tienen experiencia enseñando a haitianos declaran que este colectivo prefiere las clases más convencionales, silenciosas y concentradas. Lo importante en este punto es distinguir bien aquellas actividades que propician lo lúdico de aquellas que requieren mayor concentración, para que los estudiantes identifiquen estos momentos con claridad, sin que les parezca que toda la clase fue seria y aburrida, o lo contrario, que toda la clase fue solo juego, diversión y poca seriedad. También es conveniente que tras las actividades los profesores evidencien y destaquen lo que sus alumnos han aprendido y, por supuesto, que antes de las actividades los profesores expliciten los objetivos de estas.

Como se señaló, los aprendientes haitianos tienden a valorar mejor y a practicar más sus destrezas receptivas. En este punto vale la pena que el profesor tome el camino contrario a las preferencias de sus estudiantes, manteniendo un equilibrio prudente entre la práctica receptiva y el refuerzo de la práctica oral. La idea es que estos estudiantes pierdan el miedo a comunicarse y a cometer errores. Esto les permitiría adquirir mayor confianza en el manejo del español y, con ello, posicionarse frente a sus distintos interlocutores con un sentido de adecuación en el que la distancia social o el poder relativo no intervengan de forma negativa en la capacidad comunicativa de estos aprendientes (Norton 1995). En relación con esto mismo, sería muy conveniente la práctica de estrategias comunicativas en diversas situaciones y contextos socioculturales de la comunidad meta.

Finalmente, este cuestionario deja bastante claro que los temas que más quieren abordar los haitianos se relacionan con el trabajo y la escuela, lo que no es de extrañar considerando que en estos ámbitos el español cubre necesidades urgentes. Lo anterior no implica descuidar el tratamiento de otros temas relevantes para este colectivo, como son la vida en comunidad, la cultura y la vida cotidiana (Serafini et al. 2015a). Si bien las primeras etapas de inmersión sociocultural corresponden a la vida laboral y/o de escuela, las etapas que consagran a los inmigrantes como hablantes multiculturales tienen que ver con una amplia gama de aspectos relacionados con la sociedad y la cultura meta, a los que también debemos dar cabida en las clases de ELE, conforme avanza el nivel de lengua de nuestros aprendices (Moreno 2009). 
Como desarrollo posterior a este trabajo, sería conveniente explorar también las estrategias preferidas por esta comunidad de aprendientes, ámbito que no alcanzó a cubrir de manera más amplia nuestro análisis de necesidades.

Gloria Toledo Vega

Pontificia Universidad Católica de Chile

gtoledo@uc.cl

ORCID: 0000-0003-1031-2844

Libertad Korchabing

Escuela y Fundación Jean Jacques Dessalines

korchabing@gmail.com

Recepción: 18/11/2018; Aceptación: 19/09/2019

\section{Referencias bibliográficas}

Altman, H. B. 1980. "Foreign Language Teaching: Focus on the learner". En Foreign Language Teaching: Meeting Individual Needs, H. B. Altman y C.V. James (eds.), 116. New York: Pergamon Press.

Arancibia, M., Behar, R., Marín, S., Inzunza, N. y Madrid, E. 2016. "Funcionamiento psicosocial en pacientes agudos y crónicos no psiquiátricos en régimen hospitalario: depresión, alexitimia y falta de asertividad”. Rev Med Chile, 144 (11). 1424-1431.

Benson, P. 2001. Teaching and Researching Autonomy in Language Learning. Harlow: Pearson Education.

Benson, P. y Nunan, D. 2005. Learner's Stories: Difference and Diversity in Language Learning, Cambridge: Cambridge University Press.

Berwick, R. 1989. "Needs assessment in Language Programming: from Theory to Practice". En The Second Language Curriculum, R.K. Johnson (ed.), 48 - 62. New York: Cambridge University Press.

Brindley, C. 1989. "The Role of Needs Analysis in Adult ESL Programme Design”. En The Second Language Curriculum, R.K. Johnson (ed.), 63 - 78. New York: Cambridge University Press.

Brown, J.D. 1989. “Language program evaluation: a synthesis of existing possibilities”. En The Second Language Curriculum, R. K. Johnson (ed.), 222 - 241. Cambridge University Press.

Catalá, A. 2017. Qué Enseñar En Una Clase De Español Con Fines Específicos: El Análisis De Necesidades. Universitat De Barcelona. Departament De Filologia Hispànica. Disponible en $<$ http://hdl.handle.net/10803/458442>.

Christison, M. A. 2003. "Learning styles and strategies". En Practical English Language Teaching, Nunan, D. (Eed.), 26-288. McGraw-Hill, New York.

Cohen, A. D. 2003. “The Learner's Side of Foreign Language Learning: where do styles, strategies, and tasks meet?” International Review of Applied Linguistics, 41:4. $279-291$.

Consejo de Europa. 2000. European Language Portfolio (ELP): Principles and Guidelines. Strasbourg: Council of Europe

Departamento de Extranjería y Migración de Chile. 2018. Minuta: migración haitiana en Chile. Ministerio del Interior y Seguridad Pública de Chile.

García Santa-Cecilia, A. 2008. Cómo se diseña un curso de lengua extranjera. Madrid: Arco/Libros.

Lengua y migración / Language and Migration 12:1 (2020), 101-122

Edición impresa: ISSN 1889-5425. Edición en línea: ISSN 2660-7166. (C) Universidad de Alcalá 
García-Romeu, J. 2006. “Análisis de necesidades para la programación de cursos de fines específicos”. En Actas de III Congreso Internacional de Español para Fines Específicos, Utrecht, (ed.). 145-161 [Secretaría General Técnica de Ministerio de Educación y Ciencia]. Disponible en <http://cvc.cervantes.es/ensenanza/biblioteca_ele/ciefe/pdf/03/ cvc_ciefe_03_0014.pdf >.

Gardner, R. C. y Lambert, W. E. 1959. "Motivational variables in second language acquisition”. Canadian Journal of Psychology, 13. 266 - 272. 10.1037/h0083787.

George, D. y Mallery, P. 2003. SPSS for Windows step by step: A simple guide and reference. Boston: Allyn \& Bacon

Gliem, J. A. y Gliem, R. R. 2003. “Calculating, Interpreting, and Reporting Cronbach's Alpha Reliability Coefficient for Likert-type Scales”. En Midwest Research to Practice Conference in Adult Continuing, and Community Education. Ohio State University, Ohio.

Gnutzmann, C. 2005. "English language teaching in Germany: A reflection of the national and universal importance of English”. En Teaching English to the world: History, curriculum, and practice, G. Braine (ed.), 23-33. Mahwah, NJ: Lawrence Erlbaum

Gutiérrez, R. 2013. “La dimensión lingüística de las migraciones internacionales”. Lengua y migración, 5:2. 11-28.

Harkness, J. A. y Schoua-Glusberg, A. S. 1998. “Questionnaires in translation”. En Cross-cultural survey equivalence, J. A. Harkness (eds.), 87-126. Mannheim, Germany: ZUMA.

Hawkey, R. 1980. Syllabus Design for Specific Purposes. Projects in Material Design. ELT Documento especial. London: The British Council.

Hutchinson, T. y Waters, A. 1987. English for Specific Purposes. New York: Cambridge University Press.

Jones, B., Palinesa, R. A., Ogle, D. y Carr, E. 1987. Strategic teaching and Learning: cognitive instruction in the content areas. Alexandria, Va.: Association for Supervision and Curriculum Development.

Long, M. 2005. Second Language Needs Analysis. Cambridge: Cambridge University Press.

Mc Donough, J. 1984. ESP in Perspective: A Practical Guide. London: Jo Mc Donough.

Moreno Fernández, F. 2009. "Integración sociolingüística en contextos de inmigración: marco epistemológico para su estudio en España”. Lengua y migración, 1:1. 121-156.

Munby, J. 1978. Communicative Syllabus Design. Cambridge: Cambridge University Press.

Muñoz, C. 2000. Segundas lenguas: adquisición en el aula. Barcelona: Ariel.

Norton, B. 1995. "Social Identity, Investment, and Language Learning". TESOL Quarterly, 29:1.9-31.

Norton, B. y Toohey, K. 2001. “Changing Perspectives on Good Languages Learners”. TESOL Quarterly, 35:2 307 - 322.

Nunan, D. y Burton, J. 1985. Using Learner Data in Designing Language Courses: Workshop Guide. Adelaide: National Curriculum Resource.

Nunan, D. 1988. Syllabus Design. Oxford: Oxford University Press.

Oxford, R. L. 2003. "Language Learning Styles and Strategies: concepts and relationships”. International Review of Applied Linguistics, 41:4. 271 - 278.

Richterich, R. y J. L. Chancerel. 1980. Identifying the Needs of Adults Learning a Foreign Language. Oxford: Pergamon Press.

Richterich, R. 1983. Case Studies in Identifying Langange Needs. Oxford: Pergamon Press.

Ridell, P. G. 1991. “Analyzing Students Needs in Designing Specific Purposes Language Syllabuses”. Language Learning Journal, 3. 73 - 77.

Robinson, P.C. 1991. ESP Today, A practitioners guide. Hemel Hemstead: Practice Hall.

Savage, W. y Storer, G. 1992. “An Emergent Language Program Framework: Actively Involving Learners in Needs Analysis”. System, 20:2. 187 - 199. 
Serafini, E. J., Lake, J., y Long, M. H. 2015a. "Needs analysis for specialized learner populations: Essential methodological improvements”. English for Specific Purposes, 40. 1-16. 10.1016/j.esp.2015.05.002

Serafini, E. J. y Torres, J. 2015b. "The utility of needs analysis for non domain expert instructors in designing task-based Spanish for the professions curricula”. Foreign Language Annals, 48(3). 447-472.

Sireci S. y Padilla J. 2014. "Validating assessments: introduction to the special section". Psicothema, 26. 97-9.

Slagter, P. J. 1979. Un nivel umbral. Estrasburgo: Consejo de Europa.

Todea, L., y Demarcsek, R. 2017. "Needs Analysis for Language Course Design. A Case Study for Engineering and Business Students". Vol. 200. Institute of Physics. Disponible en <https://iopscience.iop.org/article/10.1088/1757-899X/200/1/012064>.

Toledo, G. 2016. "Propuesta didáctica para la enseñanza de español como segunda lengua a inmigrantes haitianos en Chile”. Revista Lengua y Migración, 8:1. 81 - 103.

Tudor, I. 1996. Learner-centredness as Language Education, Cambridge: Cambridge University Press.

Ungureanu, C. y Georgescu, C. A. 2012. "Learners'Strategies in Language Learning”. Procedia_Social and Behavioral Sciences, 46. 5000 - 5004.

West, R. 1994. "Needs analysis in language teaching. Language Teaching”, 27:1. 1 - 19. $10.1017 /$ S0261444800007527.

Willing, K. 1994. Learning Strategies in Adult Migrant Education. National Centre for English Language Teaching and Research: Sidney.

Willing, K. 1988. Learning Styles in Adult Migrant Education. Adelaide, National Curriculum Resource Centre.

Wong, L. y Nunan, D. 2011. "The Learning Styles and Strategies of Effective Language Learners". System, 39.144 - 163.

Yalden, J. 1987. Principles for Course Design for Language Teaching. Cambridge: Cambridge University Press. 